\title{
Structure of the RNA-dependent RNA polymerase from COVID-19 virus
}

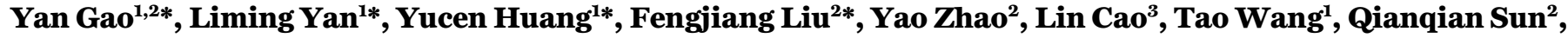 \\ Zhenhua Ming ${ }^{4}$, Lianqi Zhang', Ji Ge', Litao Zheng', Ying Zhang', Haofeng Wang ${ }^{2,5}$, Yan Zhu ${ }^{2}$, Chen Zhu' ${ }^{2}$,

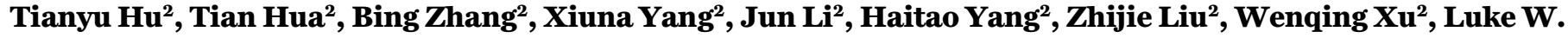

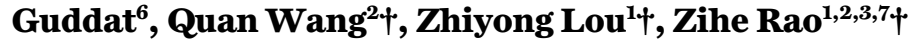 \\ ${ }^{1}$ Laboratory of Structural Biology, School of Life Sciences, and School of Medicine, Tsinghua University, Beijing, China. ${ }^{2}$ Shanghai Institute for Advanced Immunochemical \\ Studies and School of Life Science and Technology, ShanghaiTech University, Shanghai, China. ${ }^{3}$ State Key Laboratory of Medicinal Chemical Biology, Frontiers Science \\ Center for Cell Response, College of Life Sciences, and College of Pharmacy, Nankai University, Tianjin, China. ${ }^{4}$ State Key Laboratory for Conservation and Utilization of \\ Subtropical Agro-Bioresources, College of Life Science and Technology, Guangxi University, Nanning, China. ${ }^{5}$ School of Life Sciences, Tianjin University, Tianjin, China. \\ ${ }^{6}$ School of Chemistry and Molecular Biosciences, The University of Queensland, Brisbane, QLD, Australia. ${ }^{7}$ National Laboratory of Biomacromolecules, CAS Center for \\ Excellence in Biomacromolecules, Institute of Biophysics, CAS, Beijing, China.
}

*These authors contributed equally to this work.

†Corresponding author. Email: wangq@shanghaitech.edu.cn (Q.W.); louzy@mail.tsinghua.edu.cn (Z.L.); raozh@mail.tsinghua.edu.cn (Z.R.)

A novel coronavirus (COVID-19 virus) outbreak has caused a global pandemic resulting in tens of thousands of infections and thousands of deaths worldwide. The RNA-dependent RNA polymerase (RdRp, also named nsp12) is the central component of coronaviral replication/transcription machinery and appears to be a primary target for the antiviral drug, remdesivir. We report the cryo-EM structure of COVID-19 virus fulllength nsp12 in complex with cofactors nsp7 and nsp8 at 2.9- $\AA$ resolution. In addition to the conserved architecture of the polymerase core of the viral polymerase family, nsp12 possesses a newly identified $\beta$ hairpin domain at its $\mathbf{N}$ terminus. A comparative analysis model shows how remdesivir binds to this polymerase. The structure provides a basis for the design of new antiviral therapeutics targeting viral RdRp.

Corona Virus Disease 2019 (COVID-19) caused by a novel coronavirus emerged in December 2019 (1-3) and has since become a global pandemic. COVID-19 virus is reported to be a new member of the betacoronavirus genus and is closely related to severe acute respiratory syndrome coronavirus (SARS-CoV) and to several bat coronaviruses (4). Compared to SARS-CoV and MERS-CoV, COVID-19 virus exhibits faster human-to-human transmission, thus leading to the WHO declaration of a world-wide public health emergency $(1,2)$.

CoVs employ a multi-subunit replication/transcription machinery. A set of non-structural proteins (nsp) produced as cleavage products of the ORF1a and ORF1ab viral polyproteins (5) assemble to facilitate viral replication and transcription. A key component, the RNA-dependent RNA polymerase (RdRp, also known as nsp12), catalyzes the synthesis of viral RNA and thus plays a central role in the replication and transcription cycle of COVID-19 virus, possibly with the assistance of nsp7 and nsp8 as co-factors (6). Nsp12 is therefore considered a primary target for nucleotide analog antiviral inhibitors such as remdesivir, which shows potential for the treatment of COVID-19 viral infections $(7,8)$. To inform drug design we have determined the structure of nsp12, in complex with its cofactors nsp7 and nsp8 by cryo-Electron Microscopy (Cryo-EM) using two different protocols, one in the absence of DTT (Dataset-1) and the other in the presence of DTT (Dataset-2).

The bacterially expressed full-length COVID-19 virus nsp12 (residues S1-Q932) was incubated with nsp7 (residues S1-Q83) and nsp8 (residues A1-Q198), and the complex was then purified (fig. S1). Cryo-EM grids were prepared using this complex and preliminary screening revealed excellent particle density with good dispersion. After the collection and processing of 7,994 micrograph movies, we obtained a $2.9-\AA$ resolution 3D reconstruction of an nsp12 monomer in complex with one nsp7-nsp8 pair and an nsp8 monomer, as was previously observed for SARS-CoV (9). In addition to the nsp12-nsp7-nsp8 complex, we also observed single particle classes corresponding to the nsp12-nsp8 dimer, as well as individual nsp12 monomers, but these do not give atomic resolution reconstructions (fig. S2). However, the nsp12-nsp7nsp8 complex reconstruction provides the structural information for complete structural analysis.

The structure of the COVID-19 virus nsp12 contains a "right hand" RdRp domain (residues S367-F920), and a nidovirus-unique $\mathrm{N}$-terminal extension domain (residues D60-R249) that adopts a nidovirus RdRp-associated nucleotidyltransferase (NiRAN) (10) architecture. The polymerase domain and NiRAN domain are connected by an interface 
domain (residues A250-R365) (Fig. 1, A and B). An additional $\mathrm{N}$-terminal $\beta$-hairpin (D29-K50), built with the guidance of an unambiguous cryo-EM map (fig. S3A), inserts into the groove clamped by the NiRAN domain and the palm subdomain in the RdRp domain (Fig. 2). The nsp7-nsp8 pair shows a conserved structure similar to the SARS-CoV nsp7-nsp8 pair $(9,11)$. The orientation of the N-terminal helix of the separate nsp8 monomer bound to nsp12 is shifted compared with that in the nsp7-nsp8 pair (fig. S4A). The 13 additional amino acid residues resolved at the N-terminal of nsp8 show that the long "shaft" of its well-known "golf club" shape is bent (fig. S4B).

The overall architecture of the COVID-19 virus nsp12nsp7-nsp8 complex is similar to that of SARS-CoV with an rmsd value of 0.82 for $1,078 \mathrm{Ca}$ atoms (fig. S4C). However, there are key features that distinguish the two. The cryo-EM map allowed us to build the complete structure of COVID-19 virus nsp12 including all residues except S1-D3 and G897D901. In contrast the first 116 residues were not resolved in SARS-CoV nsp12 (9). The portion of the NiRAN domain resolved in SARS-CoV-12 (residues 4 to 28 and 51 to 249) comprises eight helices with a five-stranded $\beta$-sheet at the $\mathrm{N}$ terminus. (9) (Fig. 2A). In the COVID-19 virus structure we additionally resolved residues A4-R118. These constitute a structural block with five anti-parallel $\beta$-strands and two helices. Residues N215-D218 form a $\beta$-strand in COVID-19 virus nsp12 whereas these residues are less ordered in SARS-CoV nsp12. This region make contact with the strand that includes residues V96-A100, thus contributing to the stabilization of its conformation. As a result, these four strands form a compact semi $\beta$-barrel architecture. Therefore, we identify residues A4-T28 and Y69-R249 as the complete coronaviral NiRAN domain. With the resolution of N-terminal residues, we are also able to identify an N-terminal $\beta$-hairpin (D29 to K50, Figs. 1A and 2A). This $\beta$-hairpin inserts in the groove clamped by the NiRAN domain and the palm subdomain in the RdRp domain and forms a set of close contacts to stabilize the overall structure (Fig. 2B and fig. S5). Another point to note is that, we have observed C301-C306 and C487-C645 form disulfide bonds in the absence of DTT (Dataset-1). However, in the presence of DTT (Dataset-2), chelated zinc ions are present and in the same location as that observe in SARSCoV (fig. S3B).

The polymerase domain adopts the conserved architecture of the viral polymerase family (12) and is composed of three subdomains; a fingers subdomain (residues L366-A581 and K621-G679), a palm subdomain (residues T582-P620 and T680-Q815), and a thumb subdomain (residues H816-E920) (Fig. 1). The catalytic metal ions, which are observed in several structures of viral polymerases that synthesize RNA (13, 14) are not observed here in the absence of primer-template RNA and NTPs.
The active site of the COVID-19 virus RdRp domain is formed by the conserved polymerase motifs A-G in the palm domain and configured like other RNA polymerases (Figs. 1A and $3 \mathrm{~A}$ and fig. S6). Motif A comprising residues 611TPHLMGWDYPKCDRAM- 626 contains the classic divalentcation-binding residue D618, which is conserved in most viral polymerases including HCV ns5b (residue D220) and poliovirus (PV) $3 \mathrm{D}^{\text {pol }}$ (residue D233) (13, 14) (Fig. 3, B and C) and Motif C (residues 753-FSMMILSDDAVVCFN-767) contains the catalytic residues (759-SDD-761) in the turn between two $\beta$-strands. These catalytic residues are also conserved in most viral RdRps, e.g., 317-GDD-319 in HCV ns5b and 327-GDD$329 \mathrm{PV} 3 \mathrm{D}^{\mathrm{pol}}$ with the first residue being either serine or glycine.

In this structure, as in other RNA polymerases, the template/primer entry, nucleoside triphosphate (NTP) entry, and nascent strand exit paths are positively charged and solventaccessible and converge in a central cavity where the RdRp motifs mediate template-directed RNA synthesis (Fig. 3D). The configurations of the template/primer entry paths, the nucleoside triphosphate (NTP) entry channel, and the nascent strand exit path are similar to those described for SARS$\mathrm{CoV}$ and for other RNA polymerases such as HCV and PV polymerase (14) (Fig. 3, B and C). The NTP entry channel is formed by a set of hydrophilic residues, including K545, R553 and R555 in motif F. The RNA template is expected to enter the active site composed of motifs $\mathrm{A}$ and $\mathrm{C}$ through a groove clamped by motif $\mathrm{F}$ and $\mathrm{G}$. Motif $\mathrm{E}$ and the thumb subdomain support the primer strand. The product-template hybrid exits the active site through the RNA exit tunnel at the front side of the polymerase.

Remdesivir, the single $S p$ isomer of the 2-ethylbutyl L-alaninate phosphoramidate prodrug (15) (fig. S7), has been reported to inhibit COVID-19 virus proliferation and therefore have clinical potential $(7,8)$. We will briefly discuss its possible binding and inhibition mechanism based on the results of this study. The efficacy of chain-terminating nucleotide analogs requires viral RdRps to recognize and successfully incorporate the active form of the inhibitors into the growing RNA strand. Sofosbuvir (2'-F-2'-C-methyluridine monophosphate) is a prodrug which targets HCV ns5b and has been approved for the treatment of chronic HCV infection (16). It acts by binding to the catalytic site of $\mathrm{HCV}$ ns5b polymerase $(12,16)$. Given that remdesivir and sofosbuvir are both nucleotide analogs and the structural conservation of the catalytic site between COVID-19 virus nsp12 and HCV ns5b polymerase $(13,16)$ (fig. S7), we modeled remdesivir diphosphate binding to COVID-19 virus nsp12 based on superposition with sofosbuvir bound to HCV ns5b (Fig. 4A and fig. S4D). Overall, we found that the nsp12 of covid-19 virus has the highest similarity with the Apo state of ns5b. Given the conformational changes of ns $5 \mathrm{~b}$ in apo/elongation/inhibited states, it appears 
catalytic residues D760, D761 and the classic D618 will undergo a conformational change to coordinate the divalent cations (Fig. 4B). The latter will anchor the phosphate group of the incoming nucleotide or inhibitors together with the allosteric R555 in motif F (Fig. 4C). In the structures of HCV ns5b elongation complex or its complex with pp-sofosbuvir, a key feature is that the incorporated pp-sofosbuvir interacts with N291 (equivalent to N691 in COVID-19 virus) but, due to a fluorine substitution on its sugar moiety, is not capable to joint into the hydrogen bonding network with S282 and D225 (Fig. 4D), which is necessary to stabilize the incoming natural nucleotide (13). However, remdesivir keeps an intact ribose group, so it may be able to utilize this hydrogen bonds network like a native substrate. In addition, T680 in COVID-19 virus nsp12 is also likely to form hydrogen bonds with the 2' hydroxyl of Remdesivir and of course with incoming natural NTP (Fig. 4D). Moreover, the hydrophobic side chain of V557 in motif $\mathrm{F}$ is likely to stack with and stabilize the +1 template RNA uridine base to base pair with the incoming triphosphate remdesivir (ppp-remdesivir) (Fig. 4E).

The rapid global spread of COVID-19 virus has emphasized the need for the development of new coronavirus vaccines and therapeutics. The viral polymerase nsp12 looks an excellent target for new therapeutics, especially given that lead inhibitors already exist in the form of compounds such as Remdesivir. Considering the structural similarity of nucleoside analogs, the binding mode and inhibition mechanism discussed here may also be applicable to other such kind drugs or drug candidates include Favipiravir, which proves effective in clinical trials (17). This target, in addition to other promising drug targets such as the main protease, could support the development of a cocktail of anti-coronavirus treatments that potentially can be used for the discovery of broadspectrum antivirals.

\section{REFERENCES AND NOTES}

1. J. F. Chan, S. Yuan, K.-H. Kok, K. K.-W. To, H. Chu, J. Yang, F. Xing, J. Liu, C. C.-Y. Yip, R. W.-S. Poon, H.-W. Tsoi, S. K.-F. Lo, K.-H. Chan, V. K.-M. Poon, W.-M. Chan, J. D. Ip, J.-P. Cai, V. C.-C. Cheng, H. Chen, C. K.-M. Hui, K.-Y. Yuen, A familial cluster of pneumonia associated with the 2019 novel coronavirus indicating person-toperson transmission: A study of a family cluster. Lancet 395, 514-523 (2020). doi:10.1016/S0140-6736(20)30154-9 Medline

2. N. Chen, M. Zhou, X. Dong, J. Qu, F. Gong, Y. Han, Y. Qiu, J. Wang, Y. Liu, Y. Wei, J. Xia, T. Yu, X. Zhang, L. Zhang, Epidemiological and clinical characteristics of 99 cases of 2019 novel coronavirus pneumonia in Wuhan, China: A descriptive study. Lancet 395, 507-513 (2020). doi:10.1016/S0140-6736(20)30211-7 Medline

3. F. Wu, S. Zhao, B. Yu, Y.-M. Chen, W. Wang, Z.-G. Song, Y. Hu, Z.-W. Tao, J.-H. Tian, Y.-Y. Pei, M.-L. Yuan, Y.-L. Zhang, F.-H. Dai, Y. Liu, Q.-M. Wang, J.-J. Zheng, L. Xu, E. C. Holmes, Y.-Z. Zhang, A new coronavirus associated with human respiratory disease in China. Nature 579, 265-269 (2020). doi:10.1038/s41586-020-2008-3 Medline

4. P. Zhou, X.-L. Yang, X.-G. Wang, B. Hu, L. Zhang, W. Zhang, H.-R. Si, Y. Zhu, B. Li, C.L. Huang, H.-D. Chen, J. Chen, Y. Luo, H. Guo, R.-D. Jiang, M.-Q. Liu, Y. Chen, X.-R. Shen, X. Wang, X.-S. Zheng, K. Zhao, Q.-J. Chen, F. Deng, L.-L. Liu, B. Yan, F.-X. Zhan, Y.-Y. Wang, G.-F. Xiao, Z.-L. Shi, A pneumonia outbreak associated with a new coronavirus of probable bat origin. Nature 579, 270-273 (2020). doi:10.1038/s41586-020-2012-7 Medline

5. J. Ziebuhr, The coronavirus replicase. Curr. Top. Microbiol. Immunol. 287, 57-94 (2005). doi:10.1007/3-540-26765-4 3 Medline

6. L. Subissi, C. C. Posthuma, A. Collet, J. C. Zevenhoven-Dobbe, A. E. Gorbalenya, E. Decroly, E. J. Snijder, B. Canard, I. Imbert, One severe acute respiratory syndrome coronavirus protein complex integrates processive RNA polymerase and exonuclease activities. Proc. Natl. Acad. Sci. U.S.A. 111, E3900-E3909 (2014). doi:10.1073/pnas.1323705111 Medline

7. M. Wang, R. Cao, L. Zhang, X. Yang, J. Liu, M. Xu, Z. Shi, Z. Hu, W. Zhong, G. Xiao, Remdesivir and chloroquine effectively inhibit the recently emerged novel coronavirus (2019-nCoV) in vitro. Cell Res. 30, 269-271 (2020) doi:10.1038/s41422-020-0282-0 Medline

8. M. L. Holshue, C. DeBolt, S. Lindquist, K. H. Lofy, J. Wiesman, H. Bruce, C. Spitters, K. Ericson, S. Wilkerson, A. Tural, G. Diaz, A. Cohn, L. Fox, A. Patel, S. I. Gerber, L. Kim, S. Tong, X. Lu, S. Lindstrom, M. A. Pallansch, W. C. Weldon, H. M. Biggs, T. M. Uyeki, S. K. Pillai, Washington State 2019-nCoV Case Investigation Team, First Case of 2019 Novel Coronavirus in the United States. N. Engl. J. Med. 382, 929 936 (2020). doi:10.1056/NEJMoa2001191 Medline

9. R. N. Kirchdoerfer, A. B. Ward, Structure of the SARS-CoV nsp12 polymerase bound to nsp7 and nsp8 co-factors. Nat. Commun. 10, 2342 (2019). doi:10.1038/s41467-019-10280-3 Medline

10. K. C. Lehmann, A. Gulyaeva, J. C. Zevenhoven-Dobbe, G. M. C. Janssen, M. Ruben, H. S. Overkleeft, P. A. van Veelen, D. V. Samborskiy, A. A. Kravchenko, A. M. Leontovich, I. A. Sidorov, E. J. Snijder, C. C. Posthuma, A. E. Gorbalenya, Discovery of an essential nucleotidylating activity associated with a newly delineated conserved domain in the RNA polymerase-containing protein of all nidoviruses. Nucleic Acids Res. 43, 8416-8434 (2015). doi:10.1093/nar/gkv838 Medline

11. Y. Zhai, F. Sun, X. Li, H. Pang, X. Xu, M. Bartlam, Z. Rao, Insights into SARS-CoV transcription and replication from the structure of the nsp7-nsp8 hexadecamer. Nat. Struct. Mol. Biol. 12, 980-986 (2005). doi:10.1038/nsmb999 Medline

12. S. M. McDonald, RNA synthetic mechanisms employed by diverse families of RNA viruses. Wiley Interdiscip. Rev. RNA 4, 351-367 (2013). doi:10.1002/wrna.1164 Medline

13. T. C. Appleby, J. K. Perry, E. Murakami, O. Barauskas, J. Feng, A. Cho, D. Fox 3rd, D. R. Wetmore, M. E. McGrath, A. S. Ray, M. J. Sofia, S. Swaminathan, T. E. Edwards, Structural basis for RNA replication by the hepatitis $\mathrm{C}$ virus polymerase. Science 347, 771-775 (2015). doi:10.1126/science.1259210 Medline

14. P. Gong, O. B. Peersen, Structural basis for active site closure by the poliovirus RNA-dependent RNA polymerase. Proc. Natl. Acad. Sci. U.S.A. 107, 22505-22510 (2010). doi:10.1073/pnas.1007626107 Medline

15. T. K. Warren, R. Jordan, M. K. Lo, A. S. Ray, R. L. Mackman, V. Soloveva, D. Siegel, M. Perron, R. Bannister, H. C. Hui, N. Larson, R. Strickley, J. Wells, K. S. Stuthman, S. A. Van Tongeren, N. L. Garza, G. Donnelly, A. C. Shurtleff, C. J. Retterer, D. Gharaibeh, R. Zamani, T. Kenny, B. P. Eaton, E. Grimes, L. S. Welch, L. Gomba, C. L. Wilhelmsen, D. K. Nichols, J. E. Nuss, E. R. Nagle, J. R. Kugelman, G. Palacios, E. Doerffler, S. Neville, E. Carra, M. O. Clarke, L. Zhang, W. Lew, B. Ross, Q. Wang, K. Chun, L. Wolfe, D. Babusis, Y. Park, K. M. Stray, I. Trancheva, J. Y. Feng, O. Barauskas, Y. Xu, P. Wong, M. R. Braun, M. Flint, L. K. McMullan, S.-S. Chen, R. Fearns, S. Swaminathan, D. L. Mayers, C. F. Spiropoulou, W. A. Lee, S. T. Nichol, T. Cihlar, S. Bavari, Therapeutic efficacy of the small molecule GS-5734 against Ebola virus in rhesus monkeys. Nature 531, 381-385 (2016). doi:10.1038/nature17180 Medline

16. E. J. Gane, C. A. Stedman, R. H. Hyland, X. Ding, E. Svarovskaia, W. T. Symonds, R. G. Hindes, M. M. Berrey, Nucleotide polymerase inhibitor sofosbuvir plus ribavirin for hepatitis C. N. Engl. J. Med. 368, 34-44 (2013). doi:10.1056/NEJMoa1208953 Medline

17. C. Chen, J. Huang, Z. Cheng, J. Wu, S. Chen, Y. Zhang, B. Chen, M. Lu, Y. Luo, J. Zhang, P. Yin, X. Wang, Favipiravir versus Arbidol for COVID-19: A Randomized Clinical Trial. medRxiv 2020.03.17.20037432 [Preprint]. 27 March 2020. https://doi.org/10.1101/2020.03.17.20037432.

18. D. N. Mastronarde, Automated electron microscope tomography using robust prediction of specimen movements. J. Struct. Biol. 152, 36-51 (2005). doi:10.1016/i.jsb.2005.07.007 Medline

19. S. Q. Zheng, E. Palovcak, J.-P. Armache, K. A. Verba, Y. Cheng, D. A. Agard, 
MotionCor2: Anisotropic correction of beam-induced motion for improved cryoelectron microscopy. Nat. Methods 14, 331-332 (2017). doi:10.1038/nmeth.4193 Medline

20. A. Punjani, J. L. Rubinstein, D. J. Fleet, M. A. Brubaker, cryoSPARC: Algorithms for rapid unsupervised cryo-EM structure determination. Nat. Methods 14, 290-296 (2017). doi:10.1038/nmeth.4169 Medline

21. S. Li, Q. Zhao, Y. Zhang, Y. Zhang, M. Bartlam, X. Li, Z. Rao, New nsp8 isoform suggests mechanism for tuning viral RNA synthesis. Protein Cell 1, 198-204 (2010). doi:10.1007/s13238-010-0028-8 Medline

22. E. F. Pettersen, T. D. Goddard, C. C. Huang, G. S. Couch, D. M. Greenblatt, E. C. Meng, T. E. Ferrin, UCSF Chimera-A visualization system for exploratory research and analysis. J. Comput. Chem. 25, 1605-1612 (2004). doi:10.1002/icc.20084 Medline

23. P. Emsley, B. Lohkamp, W. G. Scott, K. Cowtan, Features and development of Coot. Acta Cryst. D66, 486-501 (2010). doi:10.1107/S0907444910007493 Medline

24. P. V. Afonine, R. W. Grosse-Kunstleve, N. Echols, J. J. Headd, N. W. Moriarty, M. Mustyakimov, T. C. Terwilliger, A. Urzhumtsev, P. H. Zwart, P. D. Adams, Towards automated crystallographic structure refinement with phenix.refine. Acta Cryst. D68, 352-367 (2012). doi:10.1107/S0907444912001308 Medline

\section{ACKNOWLEDGMENTS}

We would like to pay an exceptional tribute to ShanghaiTech University and their administrative team, as well as the Bio-Electron Microscopy Facility for their great care and support to our research team to enable us to carry out this research in a safe and healthy environment. It would have been impossible for us to attain this achievement without their tremendous efforts in the last two months during the COVID-19 pandemic. We also would like to convey our special thanks to Tsinghua University for their exceptional permission to allow five of $Z$. R.'s students to go back to the laboratory to prepare the protein samples for this study. We also must express our gratitude to the campus service team of ShanghaiTech University scientific research platform of Shanghai Institute for Advanced Immunochemical Studies (SIAIS), and National Center for Protein Science Shanghai (NCPSS), as well as all the manager and technician individuals those who provided onsite or remote technical support. Their kind help and fearless support are pivotal to this work during the epidemic. We would like to thank the University of Queensland and Diamond Light Source for their collaboration. Funding: This work was supported by the National Program on Key Research Project of China (grant no. 2017YFC0840300), the Strategic Priority Research Program of the Chinese Academy of Sciences (grant XDB08020200), the National Natural Science Foundation of China (grant nos. 81520108019, 813300237) and Science and Technology Commission of Shanghai Municipality (20431900200). Author contributions: Z.R. conceived, initiated, and coordinated the project. Y.H, L.Y., Yao.Z., H.W., Yan.Z., C.Z., Z.M., L.Z., J.G., L.Z., Yi.Z. and Tianyu.H. purified the protein; L.Y. supervised sample purification; Y.G., F.L., L.C., Q.S. and Tian.H. collected the cryo-EM data; Y.G. F.L. and T.W. processed cryo-EM data; Q.W., L.Y., Y.G. built and refined the structure model; and the manuscript was written by Z.L., Q.W., L.Y., Y.G., F.L., L.G. and Z.R.. All authors discussed the experiments and results, read and approved the manuscript. Competing interests: The authors declare no competing interests. Data and materials availability: The cryo-EM maps and the structures were deposited into the Electron Microscopy Data Bank (EMDB) and Protein Data Bank (PDB) with the accession numbers EMD-30127 and PDB 6M71 for Dataset-1, EMD-30178 and PDB 7BTF for Dataset-2 (under the reducing condition). This work is licensed under a Creative Commons Attribution 4.0 International (CC BY 4.0) license, which permits unrestricted use, distribution, and reproduction in any medium, provided the original work is properly cited. To view a copy of this license, visit https://creativecommons.org/licenses/by/4.0/. This license does not apply to figures/photos/artwork or other content included in the article that is credited to a third party; obtain authorization from the rights holder before using such material.

\section{SUPPLEMENTARY MATERIALS}

science.sciencemag.org/cgi/content/full/science.abb7498/DC1

Materials and Methods
Figs. S1 to S7

Table S1

References (18-24)

Movie S1

16 March 2020; accepted 7 April 2020

Published online 10 April 2020

10.1126/science.abb7498 
A

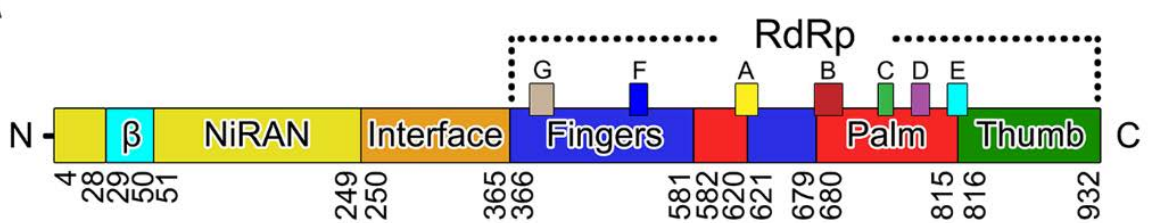

B
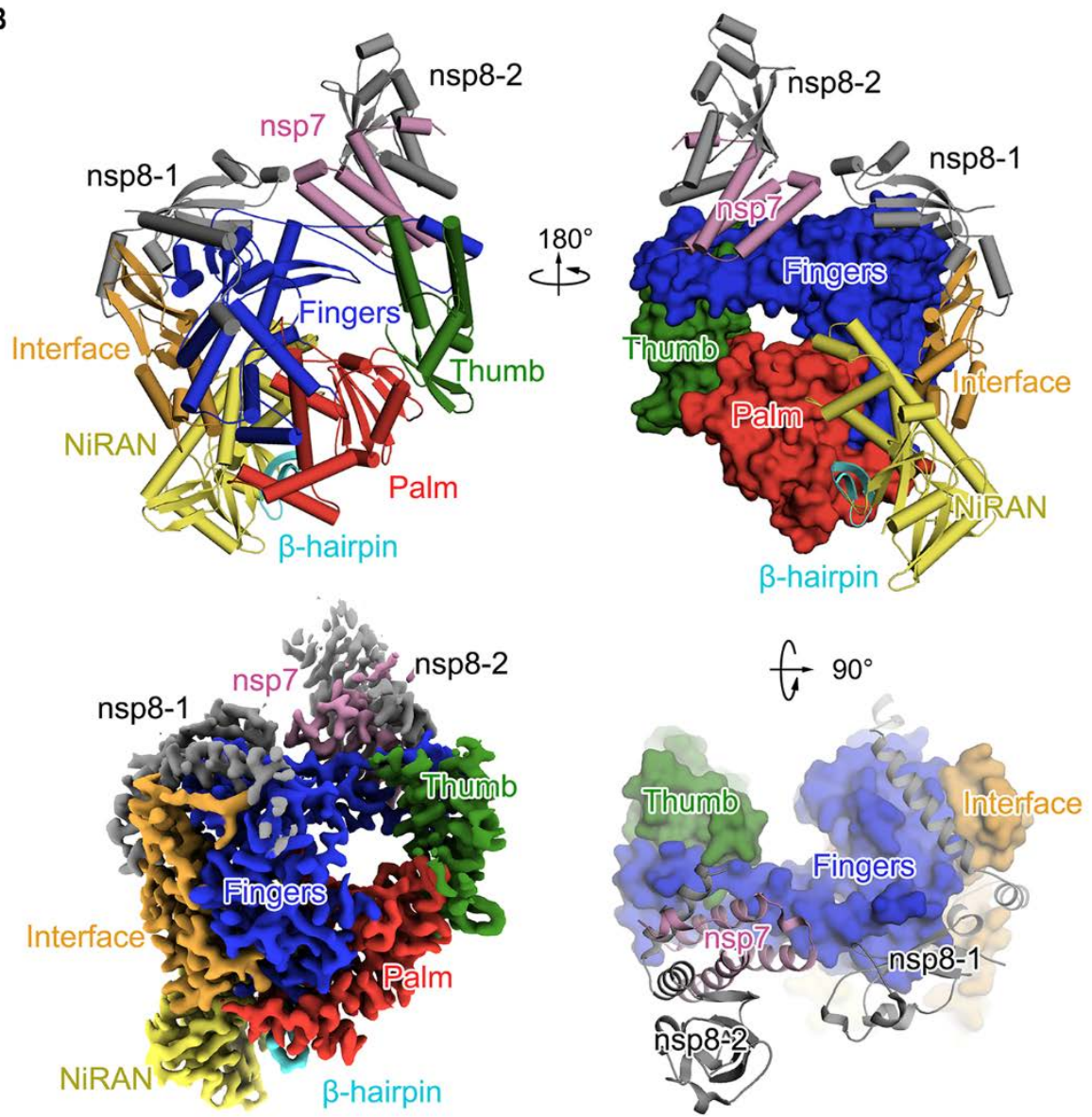

Fig. 1. Structure of COVID-19 virus nsp12-nsp7-nsp8 complex. (A) Domain organization of COVID-19 virus nsp12. The interdomain borders are labeled with residue numbers. The $\mathrm{N}$-terminal portion with no cryo-EM map density and the $\mathrm{C}$-terminal residues that cannot be observed in the map are not included in the assignment. The polymerase motifs are colored as: motif $A$, yellow; motif $B$, red; motif $C$, green; motif $D$, violet; motif $E$, cyan; motif $F$, blue; and motif G, light brown. (B) Ribbon diagram of COVID-19 virus nsp12 polypeptide chain in three perpendicular views. Domains are colored the same as in (A). The individual nsp8 (nsp8-1) bound to nsp12 and that in the nsp7-nsp8 pair (nsp8-2) are in grey; the nsp7 is in pink. The bottom left panel shows an overview of the cryo-EM reconstruction of the nsp12-nsp7nsp8 complex. 
A

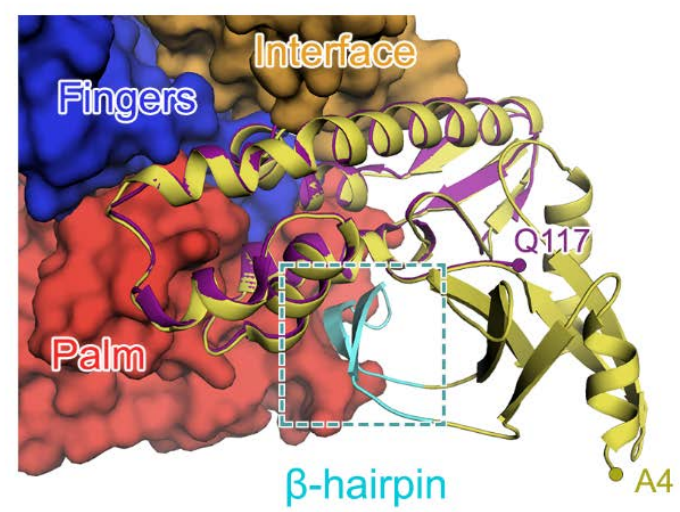

COVID-19 Virus NiRAN | SARS-CoV NiRAN
B

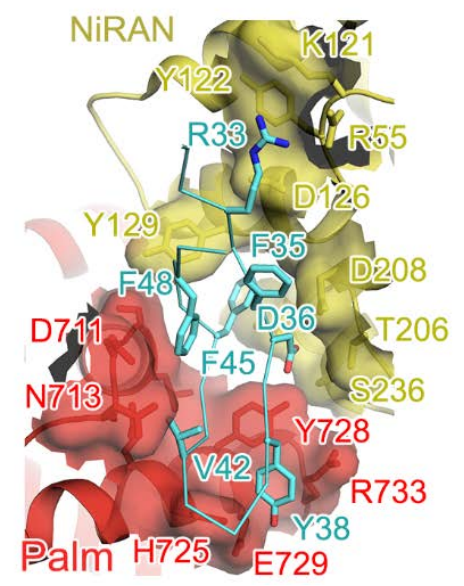

Fig. 2. Structure of $\mathrm{N}$-terminal NiRAN domain and $\beta$-hairpin. (A) Overall structure of the $N$-terminal NiRAN domain and $\beta$-hairpin of COVID-19 virus nsp12. The $\mathrm{N}$-terminal NiRAN domain and $\beta$-hairpin of COVID-19 virus nsp12 are shown as yellow and cyan cartoons, while the other regions of COVID19 virus nsp12 are shown as a molecular surface with the same color scheme used in Fig. 1. The NiRAN domain of SARS-CoV nsp12 is superimposed to its counterpart in COVID-19 virus nsp12 and is shown in purple. (B) Key interactions between the $\beta$-hairpin and other domains. The $\beta$-hairpin is shown as a cyan tube with its key residues in stick mode. These have the closest contacts with other domains of COVID-19 virus nsp12. The interacting residues in the palm and fingers subdomain of the RdRp domain, and the NiRAN domain, are identified by the labels. 
A

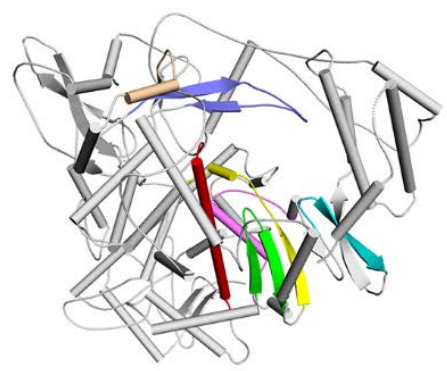

COVID-19 Virus nsp12

B

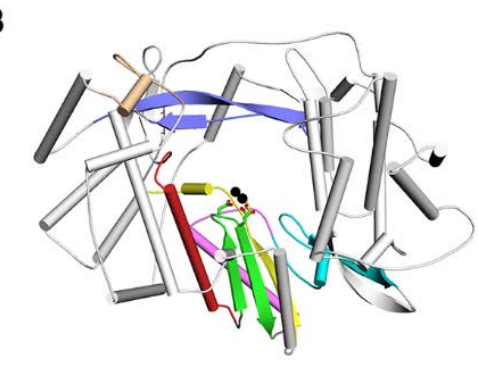

HCV ns5b

C

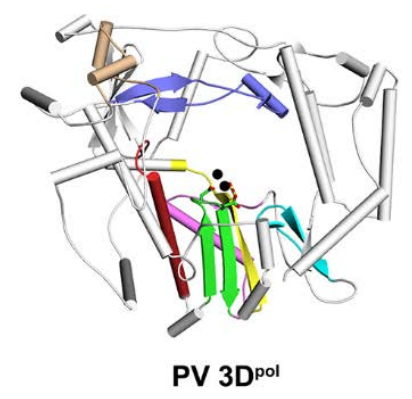

D
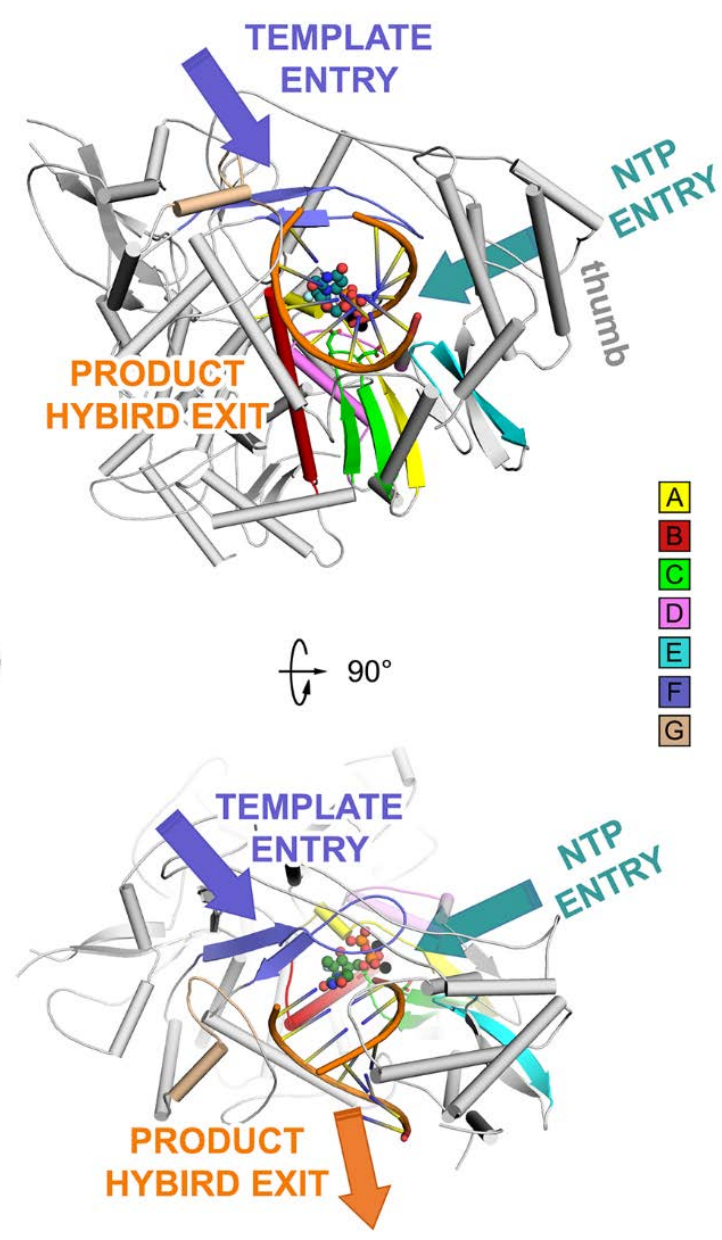

Fig. 3. The RdRp core region. (A to C) Structural comparison of COVID-19 virus nsp12 (A), HCV ns5b (PDB ID: 4WTG) (13) (B), and PV 3D pol (PDB ID: 3OLB) (14) (C). The three structures are displayed in the same orientation. The polymerase motifs $(A-G)$ have the same color scheme used in Fig. 1A. (D) The template entry, NTP entry, product hybrid exits paths in COVID-19 virus nsp12 are labeled in slate, deep teal and orange colors. Two catalytic manganese ions (black spheres), pp-sofosbuvir (dark green spheres for carbon atoms) and primer template (orange) from the structure of $\mathrm{HCV}$ ns5b in complex pp-sofosbuvir (PDB ID: 4WTG) (13) are superposed to COVID-19 virus nsp12 to indicate the catalytic site and nucleotide binding position. 
A

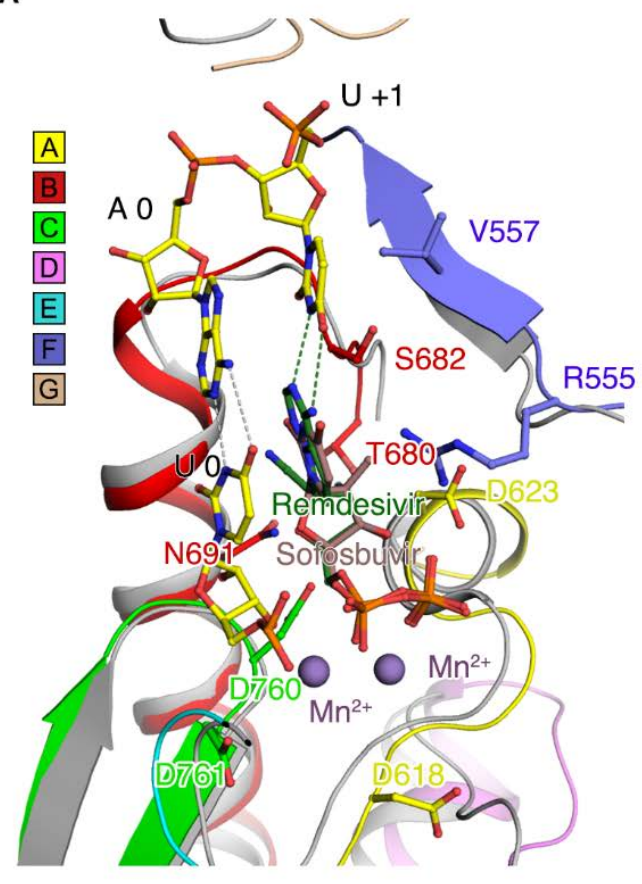

B

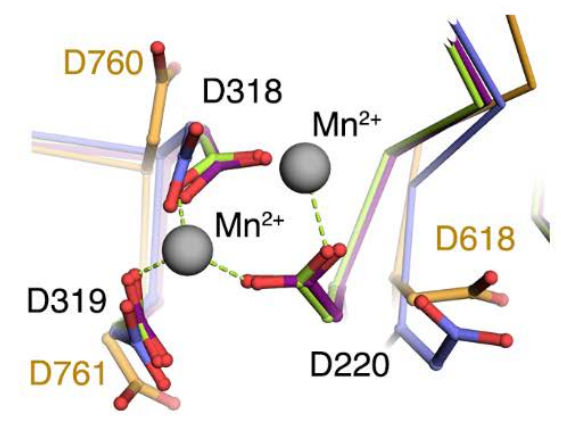

COVID-19 virus nsp12-Remdesivir
E
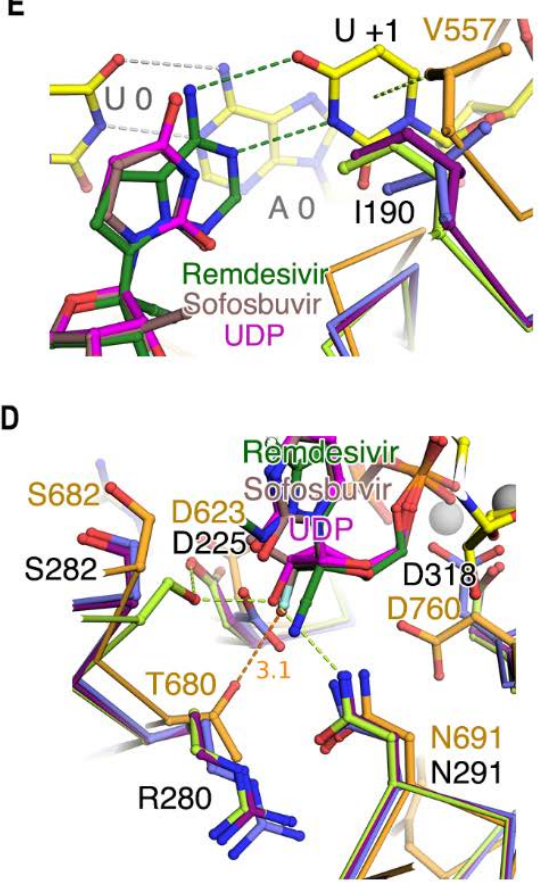

C

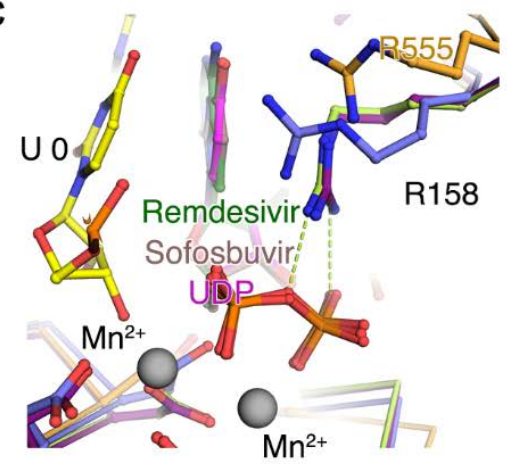

HCV ns5b-UDP

HCV ns5b-Sofosbuvir

Fig. 4. Incorporation model of remdesivir in COVID-19 virus nsp12. (A) The polymerase motifs are colored as in Fig. 3. Superposition of the structure of HCV ns5b in complex with pp-sofosbuvir (PDB ID: 4WTG) (13) with COVID-19 virus nsp12 shows the possible positions of the two catalytic ions (brown spheres), the priming nucleotide $(\mathrm{U} 0)$, template strand, and the incoming pp-remdesivir in nsp12. (B to E) Structure comparison of HCV Apo ns5b or its complex with UDP and pp-sofosbuvir with the COVID-19 virus nsp12. 